\title{
Standards and Solutions for eHealth Interoperability
}

\author{
Bernd Blobel $^{1}$, Robert Stegwee ${ }^{2}$ \\ ${ }^{1}$ eHealth Competence Center, University Hospital Regensburg, Germany \\ ${ }^{2}$ Cap Gemini, Twente, The Netherlands
}

\author{
Correspondence to: \\ Prof. Dr. Bernd Blobel \\ eHealth Competence Center \\ EJBI 2012; 8(3):1-2 \\ University Hospital Regensburg \\ Address: Franz-Josef-Strauss-Allee 11, 93053 Regensburg, Germany \\ E-mail: bernd.blobel@klinik.uni-regensburg.de
}

There is no doubt that the way to high quality, safe and efficient health services leads to distributed cooperative care (shared care) supported by information and communication technologies [1]. Such approach requires interoperability between all principals (persons, organizations, devices, applications, components) involved [1]. Nevertheless, many papers and organizations (e.g. [2]) dedicated to health care interoperability still refer to the IEEE Dictionary definition of (semantic) interoperability as "... the ability of two or more systems or components to exchange information and to use the information that has been exchanged" [3]. However, the problem of interoperability is not limited to the early days' EDI challenge of harmonized data representation and exchange protocol. Nowadays, it is the challenge of the legally, culturally, socially, educationally and organizationally impacted aspect of commonality regarding business processes and objectives, but also interests, knowledge and skills to cooperate for jointly meeting those business objectives [1]. Those aspects go far beyond ICT ontology and communication protocols as commonly understood today. Depending on the sharing of those common aspects, comprehensive interoperability can be provided at different interoperability levels such as structural, syntactic, semantic or services, semantic, or services interoperability depending on the level of shared knowledge and skills [1].

The deployment of the interoperability paradigm is even more challenging in the case of providing health services covering diagnosis and therapy, but also independent and healthy living independent of time and location of principals and resources. Thereby, the services are not limited to caring patients (health care services), but cover also prevention, social care, life style, etc., called health services addressing citizens in general. Such health service delivery over distance is the matter of eHealth. Thereby, technology paradigms such as mobile and pervasive computing as well as adaptive systems for personalization have to be exploited [1].

eHealth interoperability requires an open systems ap- proach, agreed methodologies and processes, the use of reference models and architectures, reference terminologies and ontologies, but also user acceptance and trustworthiness. The agreement on those requirements is established in international standards and specifications. For guaranteeing interoperability, the development process and implementation details must be harmonized. Here, the application of tools and the agreement on implementation guide comes into play.

There are many Standards Developing Organizations (SDOs) contributing to eHealth interoperability. Here, ISO and especially ISO TC 215 Health Informatics, CEN and especially CEN TC 251 Health Informatics, but also HL7 International, IHTSDO, OASIS, OMG, and many others have to be mentioned. For enabling practical interoperability, the more general and generic specifications have to be use case specifically profiled. This is managed by IHE.

Many of the HL7 standards and specifications covering most of the health information interoperability challenges have been meanwhile approved at ISO. The current Special Issue of the European Journal of Biomedical Informatics (EJBI) is mainly based on papers submitted to the International HL7 Interoperability Conference (IHIC) 2012, organized by HL7 Austria and performed from 27-28 September 2012 in Vienna, Austria.

The call of EJBI for the special topic volume "Standards and Solutions for eHealth Interoperability" attracted many authors for submissions. The quality of submissions was very high, which resulted in a big number of accepted papers beyond the usual number of pages for EJBI volumes despite the strict review process performed. Therefore, the editors have decided to publish the accepted submissions in two issues of EJBI - no. 3 and no. 4 - in 2012. The rest set of papers is published in no. 3 of EJBI, and its printed version will be distributed among participants of the IHIC 2012. The second part of accepted submissions will be published only electronically in no. 4 of EJBI in 2012. 
The EJBI volume at hand addresses standards and specifications and their implementation in local, regional international eHealth solutions, thereby representing different institutions, countries and regions around the globe.

In no. 3 of this EJBI Special Issue, first aspects and methodologies setting foundations for eHealth interoperability are presented. Catalina Martínez Costa, André Queiroz de Andrade, Daniel Karlsson, Dipak Kalra, and Stefan Schulz tackle in their contribution the semantic interoperability challenge when differently representing concepts using different terminologies. Edgar De la Cruz, Diego M. Lopez, and Bernd Blobel describe an architecture-centric model-driven approach for automatically harmonizing information held in different EHR systems to enable semantic interoperability based on HL7 CDA. Similarly, Frank Oemig and Bernd Blobel present an architecture-centric, ontology-driven method for automatically designing communication interfaces for workable interoperability. Philip Scott, Robert Worden, and David Bowen finally present an open source tool for easily editing clinical documents by clinicians using the HL7 CDA approach. The solution can be deployed for any CDA profile.

A series of papers addresses examples for practical health information interoperability solutions dedicated to certain specialties and/or diseases. Alberto Moreno, Alicia Martínez, Francisco Núñez, and Carlos Parra developed an infrastructure to enable the continuity of care for chronic kidney patients by sharing related information between EHR systems, which has been semantically harmonized using the openEHR Archetype approach. Patricia A. H. Williams, Sarah Gaunt, Grahame Grieve, Vincent McCauley, and Hugh Leslie demonstrate and critically discuss the use of CDA to implement shared Personally Controlled Electronic Health Records as well as to enable document exchange between healthcare establishments at national level in Australia. Carina Seerainer, Hannes Kumpusch, Michael Nitzlnader, and Günter Schreier have specified and implemented a Severe Adverse Events (SAE) report for clinical trials in the field of paediatric oncology. The solution is based on the HL7 CDA methodology using existing CDA Implementation Guides. It has been demonstrated that for comprehensive interoperability in large networks, beside information also business rules must be harmonized. Sergey Shvyrev, Maxim Surin, and Vitally Rodionov present a solution for automated creation of ultrasound notes in diagnostic pathol- ogy based on the SNOMED CT terminology. Mihaela Vida, Lacramioara Stoicu-Tivadar, Bernd Blobel, and Elena Bernad developed and practically implemented a solution for an architecture-centric framework for the modeldriven development of obstetrics-gynecology department information systems. The approach enables comprehensive semantic interoperability, covering not only shared information but also its context such as the business process.

The volume concludes with a paper addressing the pervasive aspect of eHealth. Philipp Urbauer, Matthias Frohner, Mathias Forjan, Birgit Pohn, Stefan Sauermann, and Alexander Mense have implemented and evaluated interoperability solutions between personal health devices widely used for telemonitoring patients who suffer from chronic diseases. Specifications and implementation guides are based on certified solutions provided by SDOs such as HL7 and IHE as well as the Continua Health Alliance. Despite sound technical solutions for interoperability, there are still open issues regarding legal, administrative or security and privacy aspects.

The EJBI Special Issue no. 3 at hand should be seen as an appetizer to engage the reader to looking at the full set of contributions provided electronically as well as to consult related references and project documentations. As the development is under quick move, another Special Issue on Standards and Solutions for eHealth Interoperability should follow in a few years to update the community on the addressed important field of ICT support for improving safety, quality and user-friendliness of health services and the efficiency and practicality of the related processes.

The Guest-Editors are indebted to thank all authors and reviewers for their excellent work as well as the HL7 International, HL7 Europe and especially HL7 Austria for the given logistical and financial support.

\section{References}

[1] Blobel B. Architectural Approach to eHealth for Enabling Paradigm Changes in Health. Methods Inf Med. 2010; 49(2): $123-134$

[2] Health Level Seven International (HL7 International, www.hl7.org (last access on 27 January 2012).

[3] Institute of Electrical and Electronics Engineers. IEEE Standard Computer Dictionary: A Compilation of IEEE Standard Computer Glossaries. New York, NY: 1990 